\title{
Added Prognostic Value of Cerebrospinal Fluid Biomarkers in Predicting Decline in Memory Clinic Patients in a Prospective Cohort
}

\author{
Citation for published version (APA):
}

Handels, R. L. H., Joore, M. A., Vos, S. J. B., Aalten, P., Ramakers, I. H. G. B., Rikkert, M. O., Scheltens, P., Jansen, W. J., Visser, P-J., van Berckel, B. M. N., van Domburg, P., Smid, M., Hoff, E., Hoogmoed, J., Bouwman, F., Claassen, J., Leentjens, A. F. G., Wolfs, C. A. G., Severens, J. L., \& Verhey, F. R. J. (2016). Added Prognostic Value of Cerebrospinal Fluid Biomarkers in Predicting Decline in Memory Clinic Patients in a Prospective Cohort. Journal of Alzheimer's Disease, 52(3), 875-885.

https://doi.org/10.3233/JAD-151120

Document status and date:

Published: 01/01/2016

DOI:

10.3233/JAD-151120

Document Version:

Publisher's PDF, also known as Version of record

\section{Document license:}

Taverne

Please check the document version of this publication:

- A submitted manuscript is the version of the article upon submission and before peer-review. There can be important differences between the submitted version and the official published version of record.

People interested in the research are advised to contact the author for the final version of the publication, or visit the DOI to the publisher's website.

- The final author version and the galley proof are versions of the publication after peer review.

- The final published version features the final layout of the paper including the volume, issue and page numbers.

Link to publication

\footnotetext{
General rights rights.

- You may freely distribute the URL identifying the publication in the public portal. please follow below link for the End User Agreement:

www.umlib.nl/taverne-license

Take down policy

If you believe that this document breaches copyright please contact us at:

repository@maastrichtuniversity.nl

providing details and we will investigate your claim.
}

Copyright and moral rights for the publications made accessible in the public portal are retained by the authors and/or other copyright owners and it is a condition of accessing publications that users recognise and abide by the legal requirements associated with these

- Users may download and print one copy of any publication from the public portal for the purpose of private study or research.

- You may not further distribute the material or use it for any profit-making activity or commercial gain

If the publication is distributed under the terms of Article 25fa of the Dutch Copyright Act, indicated by the "Taverne" license above, 


\title{
Added Prognostic Value of Cerebrospinal Fluid Biomarkers in Predicting Decline in Memory Clinic Patients in a Prospective Cohort
}

Ron L.H. Handels ${ }^{\mathrm{a}, \mathrm{b}, *}$, Manuela A. Joore ${ }^{\mathrm{b}, \mathrm{c}}$, Stephanie J.B. Vos ${ }^{\mathrm{a}}$, Pauline Aalten ${ }^{\mathrm{a}}$, Inez H.G.B. Ramakers ${ }^{\mathrm{a}}$, Marcel Olde Rikkert ${ }^{\mathrm{d}}$, Philip Scheltens ${ }^{\mathrm{e}}$, Willemijn J. Jansen ${ }^{\mathrm{a}}$, Pieter-Jelle Visser ${ }^{\mathrm{a}, \mathrm{e}}$, Bart M.N. van Berckele ${ }^{\mathrm{e}}$, Peter van Domburg ${ }^{\mathrm{f}}$, Machiel Smid ${ }^{\mathrm{g}}$, Erik Hoff ${ }^{\mathrm{h}}$, Jan Hoogmoed ${ }^{\mathrm{i}, \mathrm{j}}$, Femke Bouwman ${ }^{\mathrm{e}}$, Jurgen Claassen ${ }^{\mathrm{d}}$, Albert F.G. Leentjens ${ }^{\mathrm{a}}$, Claire A.G. Wolfs ${ }^{\mathrm{a}}$, Johan L. Severens ${ }^{\mathrm{k}}$ and Frans R.J. Verhey ${ }^{\mathrm{a}}$

${ }^{a}$ Alzheimer Centre Limburg, Department of Psychiatry and Neuropsychology, School for Mental Health and Neuroscience (MHeNS), Maastricht University, Maastricht, The Netherlands

${ }^{\mathrm{b}}$ CAPHRI School for Public Health and Primary Care, Faculty of Health Medicine and Life Sciences, Department of Health Organization, Policy and Economics, Maastricht University, Maastricht, The Netherlands

${ }^{\mathrm{c} D e p a r t m e n t}$ of Clinical Epidemiology and Medical Technology Assessment, Maastricht University Medical Centre, Maastricht, The Netherlands

${ }^{\mathrm{d}}$ Radboudumc Alzheimer Centre, Department of Geriatrics, Donders Centre for Brain, Cognition and Behavior, Radboud University Medical Centre, Nijmegen, The Netherlands

${ }^{\mathrm{e}}$ Department of Neurology and Alzheimer Centre, VU University Medical Centre, Amsterdam, The Netherlands

${ }^{\mathrm{f}}$ Department of Neurology, Zuyderland Medical Centre, Sittard, The Netherlands

${ }^{\mathrm{g}}$ Department of Geriatrics, Zuyderland Medical Centre, Sittard, The Netherlands

${ }^{\mathrm{h}}$ Department of Neurology, Zuyderland Medical Centre, Heerlen, The Netherlands

${ }^{i}$ Department of Geriatrics, Laurentius Hospital Roermond, Roermond, The Netherlands

${ }^{\mathrm{j}}$ Department of Geriatrics, St. Jans Gasthuis Weert, Weert, The Netherlands

${ }^{\mathrm{k}}$ Institute of Health Policy and Management, Erasmus University Rotterdam, Rotterdam, The Netherlands

Accepted 15 February 2016

\begin{abstract}
.
Background: Limited information is available on short-term prognosis of Alzheimer's disease (AD) biomarkers in cerebrospinal fluid (CSF) in addition to routine diagnostic workup.

Objective: This study aims to investigate the added prognostic value of AD CSF biomarkers.

Methods: In a prospective cohort study, clinical experts predicted cognitive and functional symptoms in 114 memory clinic patients by assessing comprehensive routine diagnostic test information (patient history, and physical, neurological, psychiatric, neuropsychological, and MRI examinations), without and with CSF biomarkers. The reference standard was the 'observed clinically relevant decline' using baseline and 1- and 2-year follow-up information.
\end{abstract}

\footnotetext{
*Correspondence to: Ron L.H. Handels, Department of Psychiatry and Neuropsychology, Maastricht University/Alzheimer Centre Limburg, P.O. Box 616, 6200 MD
}

Maastricht, The Netherlands. Tel.: +31 43 3881041; E-mail: ron.handels@maastrichtuniversity.nl. 
Results: Decline over a 2-year period was observed in 51\% of all participants (3\% in SMC, $48 \%$ in MCI, $90 \%$ in mild dementia). In the total sample, the accuracy of predicted decline did not differ significantly between routine assessment without (79\% correctly predicted) and with (74\% correctly predicted) CSF biomarkers. Subgroup analyses revealed 25 (83\%) correct predictions in SMC, 30 (68\%) in MCI, and 35 (88\%) in dementia without the use of CSF; and 21 (70\%), 27 $(61 \%)$, and $36(90 \%)$, respectively, with the use of CSF in addition to the routine assessment.

Conclusion: AD CSF biomarkers did not increase accuracy of 2-year prognosis of cognitive and functional decline when added to routine diagnostic workup. This suggests that the standard diagnostic workup without CSF biomarkers allows fairly accurate predictions for the short-term course of symptoms. Routine AD biomarkers in CSF have limited prognostic value over 2 years in persons with a suspected cognitive disorder.

Keywords: Alzheimer's disease, cerebrospinal fluid, memory disorders, mild cognitive impairment, prognosis, sensitivity and specificity

\section{INTRODUCTION}

Accurate prognosis for patients with memory complaints is considered essential for timely access to treatment, advice, and care [1]. In recent decades much research has focused on the use of biomarkers for the diagnosis of neurodegenerative disorders, for instance using amyloid- $\beta(\mathrm{A} \beta)$ and tau biomarkers in the cerebrospinal fluid (CSF) for the diagnosis of Alzheimer's disease (AD). New diagnostic research criteria for $A D$ [2-4] indicate that the use of these and other biomarkers can support the underlying etiology of cognitive impairment. AD biomarkers may also have prognostic value, but limited information is yet available on whether AD biomarkers are useful for short-term prognosis in addition to routine diagnostic workup [2,3].

Previous studies reported that CSF biomarkers increased the accuracy of predicted decline in participants with mild cognitive impairment (MCI) in addition to clinical characteristics [5-9]. However, these studies were limited in that they represented routine assessment by a single or a few measures, such as a neuropsychological test or MRI, and did not evaluate populations with subjective memory complaints (SMC) and mild dementia. The purpose of the present study was to investigate the added value of CSF biomarkers for the prediction of clinically relevant decline over a 2 -year period in a memory clinic population. To this end, we compared the prognostic value of routine diagnostic workup (consisting of patient history, and physical, neurological, psychiatric, neuropsychological, and MRI examinations) without and with CSF biomarkers in a mixed population of persons consecutively referred to a memory clinic with SMC, MCI, or mild dementia.

\section{METHODS}

\section{Participants}

This study was performed using the patient cohort of the LEARN study, which aims to assess the clinical and economic value of biomarkers for an early diagnosis of AD (NCT01450891 [10]). This cohort included 304 consecutive patients from four Dutch university memory clinics (Maastricht University Medical Centre, VU University Medical Centre, Radboud University Medical Centre, and Leiden University Medical Centre), who had been referred for a suspected cognitive disorder between 2008 and 2012. Eligibility criteria were: a Mini-Mental State Examination (MMSE) $\geq 20$; a Clinical Dementia Rating scale score $\leq 1$; availability of a reliable informer or proxy; and absence of neurological or psychiatric pathology other than neurodegenerative disorders associated with cognitive impairments. Written informed consent was obtained from all patients and their informal caregivers. The Medical Ethics Committee of Maastricht University Medical Centre approved the study. Measurements were taken at baseline, 1- and 2-year follow-up. We selected 114 participants whose data on CSF biomarkers were available, as well as clinical data from at least one follow-up measurement at 1 or 2 years after the baseline measurement.

\section{Study design}

Clinical decision making was simulated in a prospective study. Multiple trios of clinical experts (MO, PD, MS, EH, JH, FB, JC, AL, and FV) tried to reach consensus on a prognosis without using CSF biomarker information (referred to below as 
"routine assessment without CSF biomarkers") and on a prognosis in which CSF biomarker information was available to them (referred to below as "routine assessment with CSF biomarkers"). They relied on patient descriptions offering comprehensive information about the routine diagnostic test outcomes of the study participants from the baseline measurement only. Each trio was composed of experts from three different disciplines (geriatrician, old age psychiatrist, and neurologist) with at least 5 years of clinical experience in a memory clinic. Experts were not obliged to apply strict decision rules but were recommended to use diagnostic guidelines as they would do in clinical practice. Experts were asked to individually predict the course of symptoms by answering the following question as either "stable/improvement" or "clinically relevant decline": "What will be the most likely course of cognitive or daily functioning of this patient within the next 2 years?" In case of any discrepant judgments between the experts within a trio, a consensus meeting was organized in which they were invited to offer their arguments and reach consensus on the prognosis. First, the routine assessment without CSF biomarker information and the reference standard (explained further in the text) were performed. On average 8.5 months later the routine

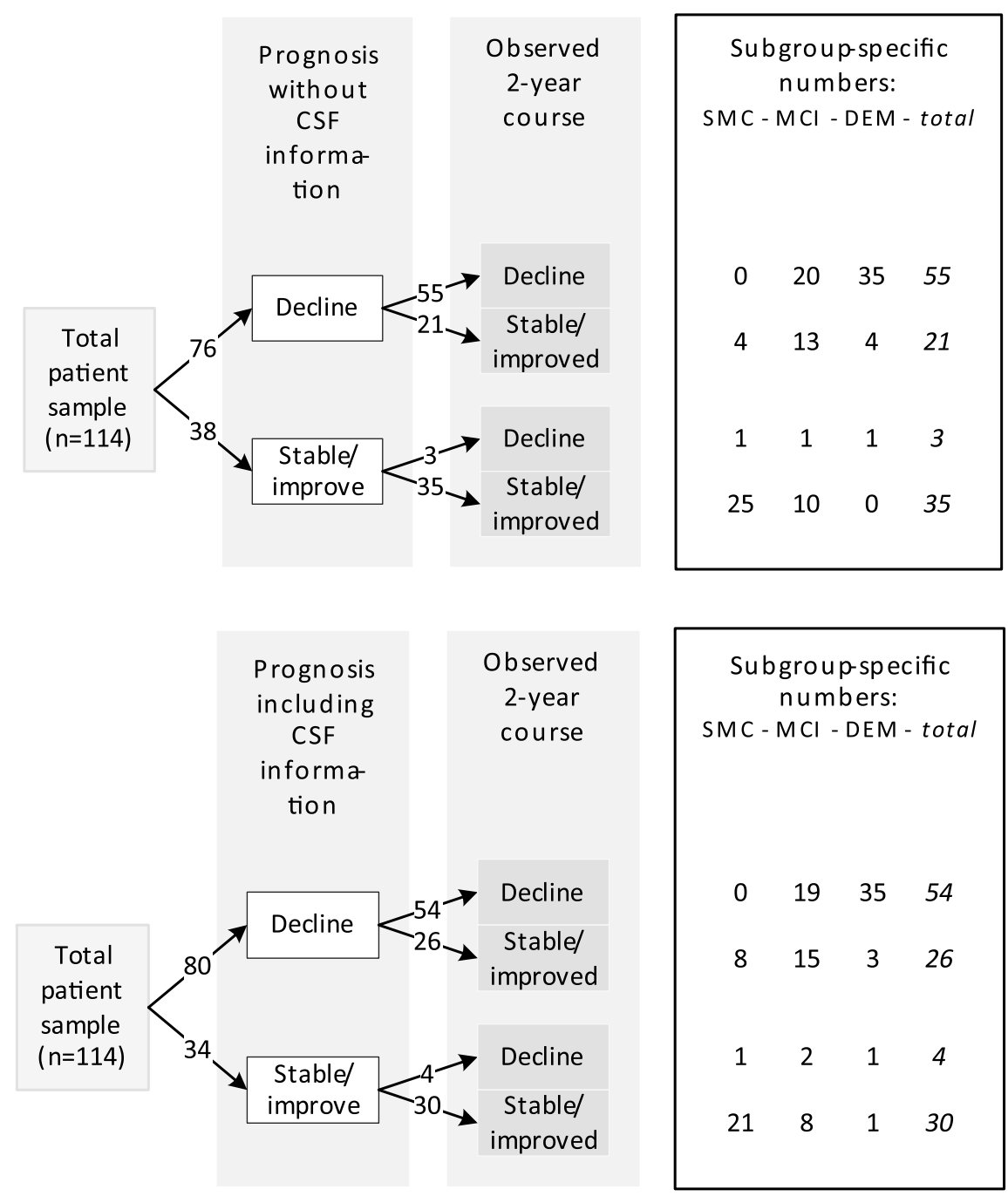

Fig. 1. Flowchart representing the prognosis based on the "routine assessment without CSF" information (upper) and the "routine assessment with CSF" information (lower) compared to the observed 2-year course of symptoms (the reference standard). SMC, subjective memory complaints; MCI, mild cognitive disorder; DEM, dementia. 
assessment with CSF biomarker information was performed to prevent experts from remembering the follow-up information, discussions, or ratings from the reference standard. Experts did not take notes during any of the assessments (see Supplementary Figure 1 and Supplementary Table 1). All assessments for each case were performed by the same trio of experts. The number of cases in which the expert and the participant originated from the same clinical center was minimized to prevent experts from recognizing a case. However, this could not be fully prevented, which resulted in one case recognized by one expert.

\section{Routine assessment and biomarker information at baseline}

The patient descriptions available to the experts included a full written history of the patient, physical, neurological, psychiatric, neuropsychological, and MRI information. Clinical information included total scores on the MMSE for global cognition [11], Clinical Dementia Rating scale for global functioning [12, 13], 15-item Geriatric Depression Scale (GDS15) for depressive symptoms [14], Neuropsychiatric Inventory for neuropsychiatric symptoms (NPI) [15], and Disability Assessment Dementia (DAD) for functioning in daily life activities [16]. The neuropsychological examination consisted of a battery of cognitive tests, harmonized across centers [10]. Tests included the 15-Word Verbal Learning Test [17, 18], Visual Association Test [19], and Digit-Span [20] to assess memory; the Letter Digit Substitution Test [21] to assess information processing speed; and the Stroop Color-Word Test [22] and Trail Making Test $[23,24]$ to assess attention, concentration, and executive functioning. Neuroimaging data were obtained at baseline only from 3-tesla MR images using a standardized protocol applied in each of the four centers. It included the medial temporal lobe atrophy rating on a visual rating scale [25], white matter lesions scored on the Fazekas scale [26], number of cortical infarcts, lacunar infarcts, and micro-bleeds, and a written description by the radiologist in case of any particularities. An example of a patient description can be found in the additional files of the publication by Handels et al. [27].

CSF information included levels of $A \beta_{1-42}$, total tau, and phosphorylated tau on a continuous scale in combination with stating the lab-specific cut-off scores $\left(\leq 550\right.$ for $A \beta_{1-42},>375$ for total tau and $>52$ for phosphorylated tau). CSF was collected at each center at the participant's baseline measurement via a lumbar puncture in the L3/L4 or L4/L5 or L5/S1 intervertebral space. CSF was centrifuged, stored at each center at $-80^{\circ} \mathrm{C}$ in polypropylene tubes, and transported on dry ice to the Department of Clinical Chemistry of VUmc Amsterdam for analysis using the same batch of reagents. CSF levels of $\mathrm{A} \beta_{1-42}$, total tau, and phosphorylated tau were measured by commercially available sandwich ELISAs (Innotest $A \beta_{1-42}$; Innotest hTAU-Ag; Innogenetics, Ghent, Belgium), blinded for all routine diagnostic workup data. Reasons for not performing a lumbar puncture $(n=172)$ were refusal by the patient or lumbar puncture being considered too burdensome by the physician. Compared to participants with CSF samples, those without CSF biomarkers were more often female $(44 \%$ versus $29 \% ; p=0.008)$ and had a higher probability of decline, though did not differ regarding other characteristics (see Supplementary Table 2).

\section{Reference standard}

The predicted course of symptoms, based on the routine assessments with and without CSF biomarkers, was verified against the observed 2-year follow-up data regarding the course of symptoms. This clinically observed course of symptoms was the "reference standard." The reference values were determined by the same trios of clinical experts. They relied on the baseline patient descriptions (patient history, physical, neurological, psychiatric, neuropsychological, and MRI information) and the descriptions from the 1- and 2-year follow-up regarding the same items except for MRI (because a scan was performed only at the participant's baseline measurement). The trios of clinical experts were blinded for any CSF biomarker information. They were asked to individually judge the observed 2-year course of symptoms by answering the following question: "In your opinion, what has been the course of cognitive or daily functioning during the 2-year follow-up period?" The answering options were limited to "stable/improvement" or "clinically relevant decline." In case of any discrepant judgments between the experts, a consensus procedure was applied similar to that for the predicted course of symptoms. More details on the expert panel method used in this study can be found elsewhere [27]. All published recommended options for designing a panel diagnosis [28] were taken into account except evaluating test-retest validity and the validity by comparing the panel diagnosis to other possible reference standards. 


\section{Statistical analyses}

Sensitivity, specificity, positive predictive value, and negative predictive value, including Wilson $95 \%$ confidence interval, were calculated for the predicted course of symptoms based on the routine assessment without CSF biomarkers, by relating them to the reference standard, as well as for the predicted course of symptoms based on the routine assessment with CSF biomarkers, by relating them to the same reference standard. The primary outcome measure was the percentage of participants for whom the predicted course of symptoms corresponded to the reference standard (i.e., the observed course of symptoms). The significance of this difference was analyzed using McNemar test in STATA12 $(p<0.05)$. Power calculation revealed a minimal detectable significant difference in the proportion of correct predictions of $9.5 \%$ (based on $80 \%$ power). We also calculated the percentage of predictions that differed between the assessment without and with CSF biomarkers (that is, participants with a predicted course of decline based on the routine assessment without CSF biomarkers versus a predicted stable/improved course based on the routine assessment with CSF biomarkers, or vice versa). The percentages of correctly differently predicted and incorrectly differently predicted courses were calculated for the assessments without and with CSF. Outcomes are presented for the total sample and for the SMC, MCI, and dementia subgroups.

\section{RESULTS}

Participants who had no CSF biomarkers available due to either unwilling to undergo a lumbar puncture, a medical contra-indication for a lumbar puncture or a failed lumbar puncture $(n=172)$, died $(n=2)$, or were unwilling to further participate $(n=16)$ before the first follow-up measurement were excluded from the analysis, leaving 114 participants for analyses. Of those 114, 89 participants had data available from both the 1- and 2-year follow-up measurement. For 19 participants, only data from the 1-year follow-up measurement was available (reasons for not performing a 2-year follow-up measurement: 4 died, 1 was admitted to institutionalized care and 6 were unwilling or untraceable, 8 were interviewed by telephone for symptom screening due to unwillingness for a full follow-up measurement). Only data from the 2-year follow-up measurement was available for 6 participants (reasons for not performing a 1-year follow-up measurement: 5 were unwilling or untraceable and 1 was interviewed by telephone for symptom screening due to unwillingness for a full follow-up measurement). These 25 participants ( $22 \%$ of the final sample) remained in the analysis. Average follow-up time was 1.83 years.

The 114 participants were aged 45-89 (mean= 67.3, SD = 8.6) years and had an average MMSE score of $26.4(\mathrm{SD}=2.9)$ and an average DAD score of $86 \%(\mathrm{SD}=16) ; 30$ of them had SMC, $44 \mathrm{MCI}$, and 40 mild dementia. See Table 1 for an overview of the baseline measurement sample characteristics by baseline syndrome diagnosis as rated by the experts. In the total sample, 58 participants (51\%) were assessed as showing clinically relevant cognitive or functional decline over the 2-year period, which reflects the reference values (3\% in SMC, $48 \%$ in MCI, $90 \%$ in mild dementia). The experts were unable to reach consensus on the prognosis for 2 participants and on the reference value for 1 participant. A majority decision was adopted for these participants.

\section{Prognosis in the assessment without and with CSF}

The prognosis based on the routine assessment without CSF biomarkers is graphically presented in the upper part of Fig. 1, together with the reference values (that is, the reference standard of the observed 2-year course of symptoms). The 2-year course of symptoms was correctly predicted for 25 (83\%) participants with SMC, 30 (68\%) with MCI, and 35 $(88 \%)$ with dementia. Based on the routine assessment with CSF biomarkers (lower part of Fig. 1), the course of symptoms was correctly predicted for 21 (70\%) participants with SMC, 27 (61\%) with MCI, and $36(90 \%)$ with dementia.

In the total sample, the proportion of correctly predicted participants did not differ significantly $(p=0.210)$ between the routine assessments without (79\%) and with (74\%) CSF biomarkers (see Table 2). After inclusion of the CSF biomarkers, the experts agreed upon a different prognosis for $16(14 \%)$ of the participants. This change was correct for 5 of them (31\%, see Table 2), 2 SMC, 1 MCI and 2 dementia patients (see Figs. 2 and 3).

In the total sample, the routine assessment without CSF biomarkers had a sensitivity of $95 \%$ (CI: 86-98), a specificity of $63 \%$ (CI: 49-74), a positive predictive value of $72 \%$ (CI: $61-81$ ), and a negative predictive value of $92 \%$ (CI: 79-97). In the total sample, the routine assessment with CSF biomarkers 


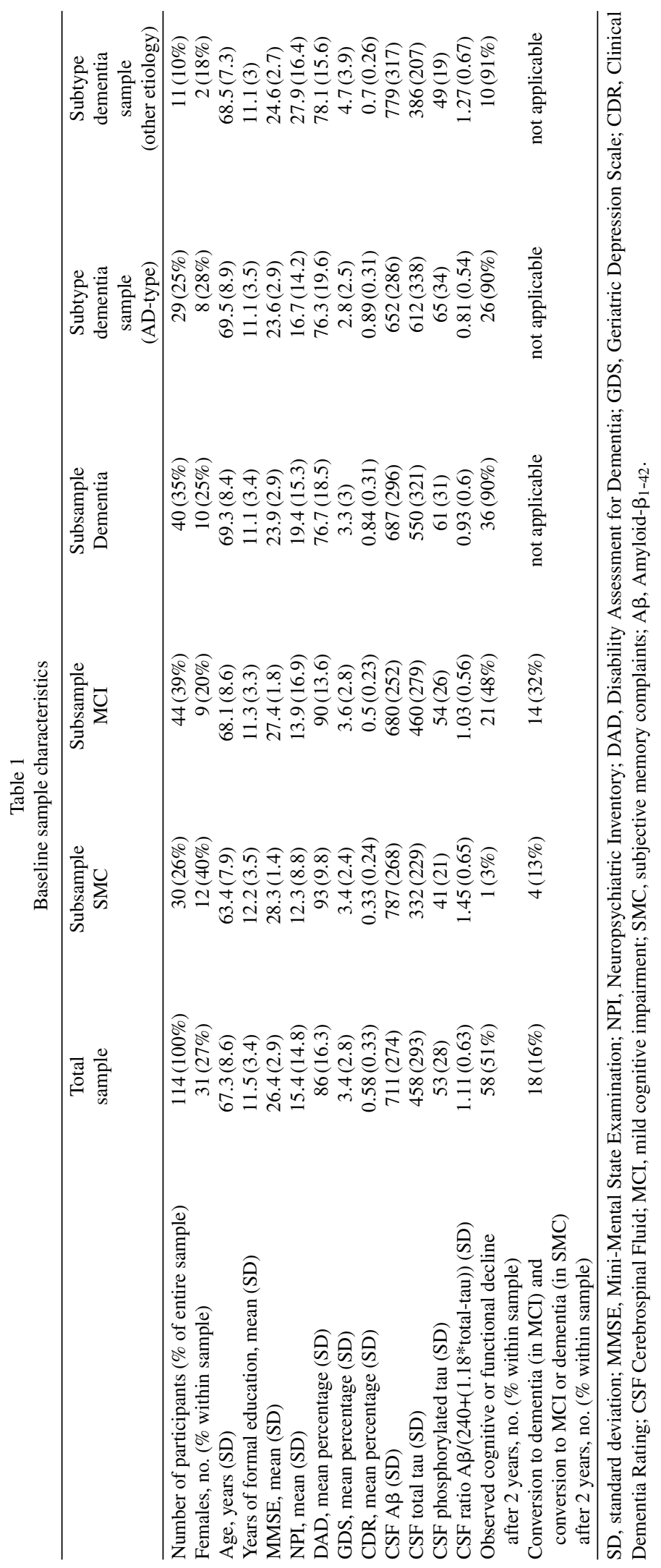


Table 2

Accuracy of the prognoses based on the "routine assessment without CSF" and the "routine assessment with CSF"

\begin{tabular}{llllccccc}
\hline Subgroup & $\begin{array}{l}\text { Routine } \\
\text { assessment }\end{array}$ & $\begin{array}{c}\text { Sensitivity } \\
\text { Wilson } \\
(95 \% \mathrm{CI})\end{array}$ & $\begin{array}{c}\text { Specificity } \\
\text { Wilson } \\
(95 \% \mathrm{CI})\end{array}$ & $\begin{array}{c}\text { Positive } \\
\text { predictive } \\
\text { value Wilson } \\
(95 \% \text { CI) }\end{array}$ & $\begin{array}{c}\text { Negative } \\
\text { predictive } \\
\text { value Wilson } \\
(95 \% \text { CI) }\end{array}$ & $\begin{array}{c}\text { Correctly } \\
\text { predicted (\%) }\end{array}$ & $\begin{array}{c}\text { Differently } \\
\text { predicted }(\%)\end{array}$ & $\begin{array}{c}\text { Of which } \\
\text { correctly } \\
\text { differently } \\
\text { predicted (\%) }\end{array}$ \\
\hline Total sample & Without CSF & $95(86-98)$ & $63(49-74)$ & $72(61-81)$ & $92(79-97)$ & $79 \%(71-85)$ & $14 \%$ & $69 \%$ \\
$(n=114)$ & With CSF & $93(84-97)$ & $54(41-66)$ & $68(57-77)$ & $88(73-95)$ & $74 \%(65-81)$ & $14 \%$ & $31 \%$ \\
SMC & Without CSF & $0^{1}(0-79)$ & $86(69-95)$ & $0(0-49)$ & $96(81-99)$ & $83 \%(66-93)$ & $27 \%$ & $75 \%$ \\
$(n=30)$ & With CSF & $0^{1}(0-79)$ & $72(54-85)$ & $0(0-32)$ & $95(78-99)$ & $70 \%(52-83)$ & $27 \%$ & $25 \%$ \\
MCI & Without CSF & $95(77-99)$ & $43(26-63)$ & $61(44-75)$ & $91(62-98)$ & $68 \%(53-80)$ & $11 \%$ & $80 \%$ \\
$(n=44)$ & With CSF & $90(71-97)$ & $35(19-55)$ & $56(39-71)$ & $80(49-94)$ & $61 \%(47-74)$ & $11 \%$ & $20 \%$ \\
Dementia & Without CSF & $97(86-100)$ & $0^{1}(0-49)$ & $90(76-96)$ & $0^{1}(0-79)$ & $88 \%(74-95)$ & $8 \%$ & $33 \%$ \\
$(n=40)$ & With CSF & $97(86-100)$ & $25^{1}(5-70)$ & $92(79-97)$ & $50^{1}(9-91)$ & $90 \%(77-96)$ & $8 \%$ & $67 \%$ \\
\hline
\end{tabular}

${ }^{1}$ Statistic was based on less than 10 participants. Differently predicted is the percentage of all participants for whom a different course was predicted from the routine assessments without and with CSF. Correctly differently predicted is the percentage of the differently predicted participants for whom the expected course was correct according to the reference standard. MCI, mild cognitive impairment; SMC, subjective memory complaints; CSF, cerebrospinal fluid.

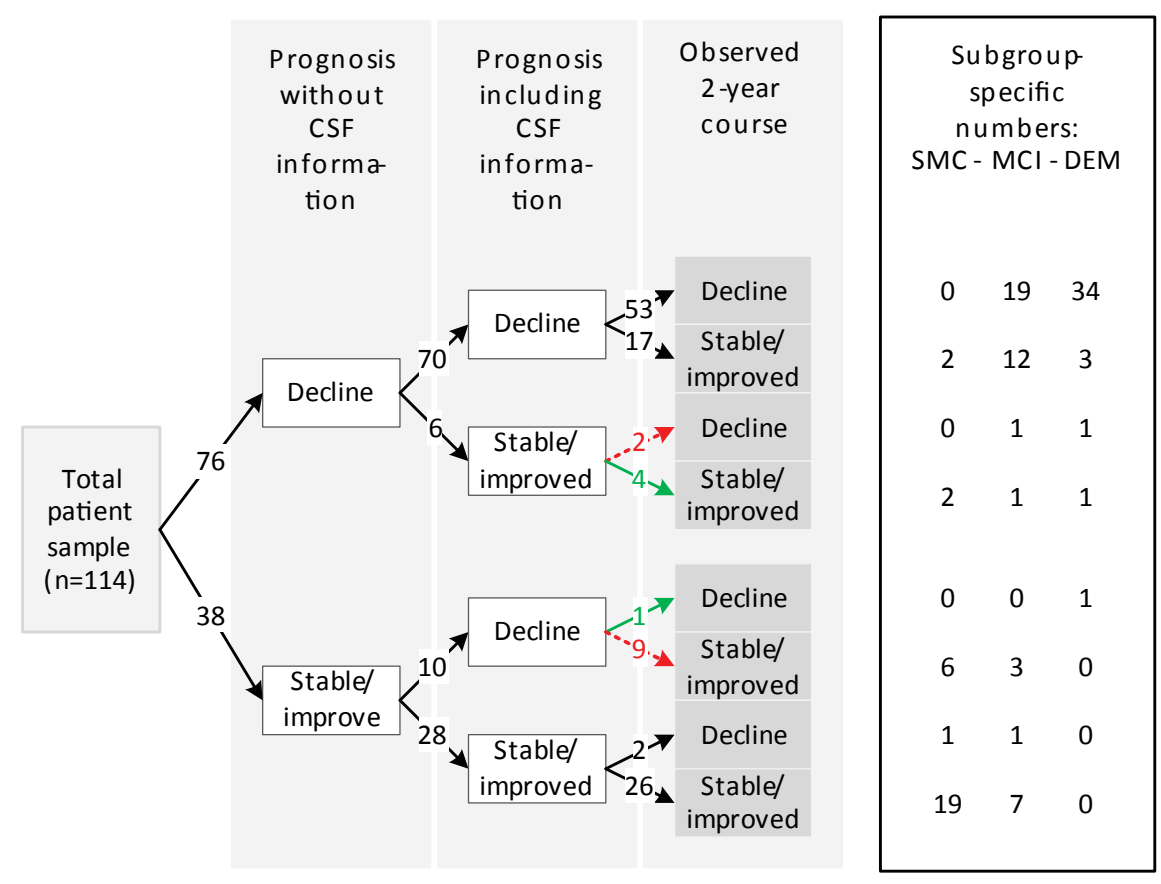

Fig. 2. Flowchart representing the prognosis based on the "routine assessment without CSF" information and the reclassification based on the "routine assessment with CSF" information, and the correctness of this reclassification (i.e. the reference standard of observed 2-year course of symptoms). SMC, subjective memory complaints; MCI, mild cognitive disorder; DEM, dementia.

sensitivity remained essentially the same (93\%; CI: 84-97), the specificity decreased (54; CI: 41-66), and both the positive predictive value (68; CI: $57-77)$, and negative predictive value were slightly lower (88; CI: 73-95). In SMC (see Table 2), sensitivity, PPV and NPV changed less than $2 \%$ while specificity decreased (-14\%). In MCI sensitivity, specificity, PPV, and NPV all somewhat decreased (between -5 to $-11 \%)$. In demented participants specificity
$(+25 \%)$ and NPV (+50\%) improved (+50\%), and sensitivity and PPV changed less than $3 \%$.

\section{DISCUSSION}

In this study, CSF biomarkers did not have an added prognostic value to routine diagnostic workup in predicting clinically relevant cognitive or functional decline within 2 years after an initial visit to 


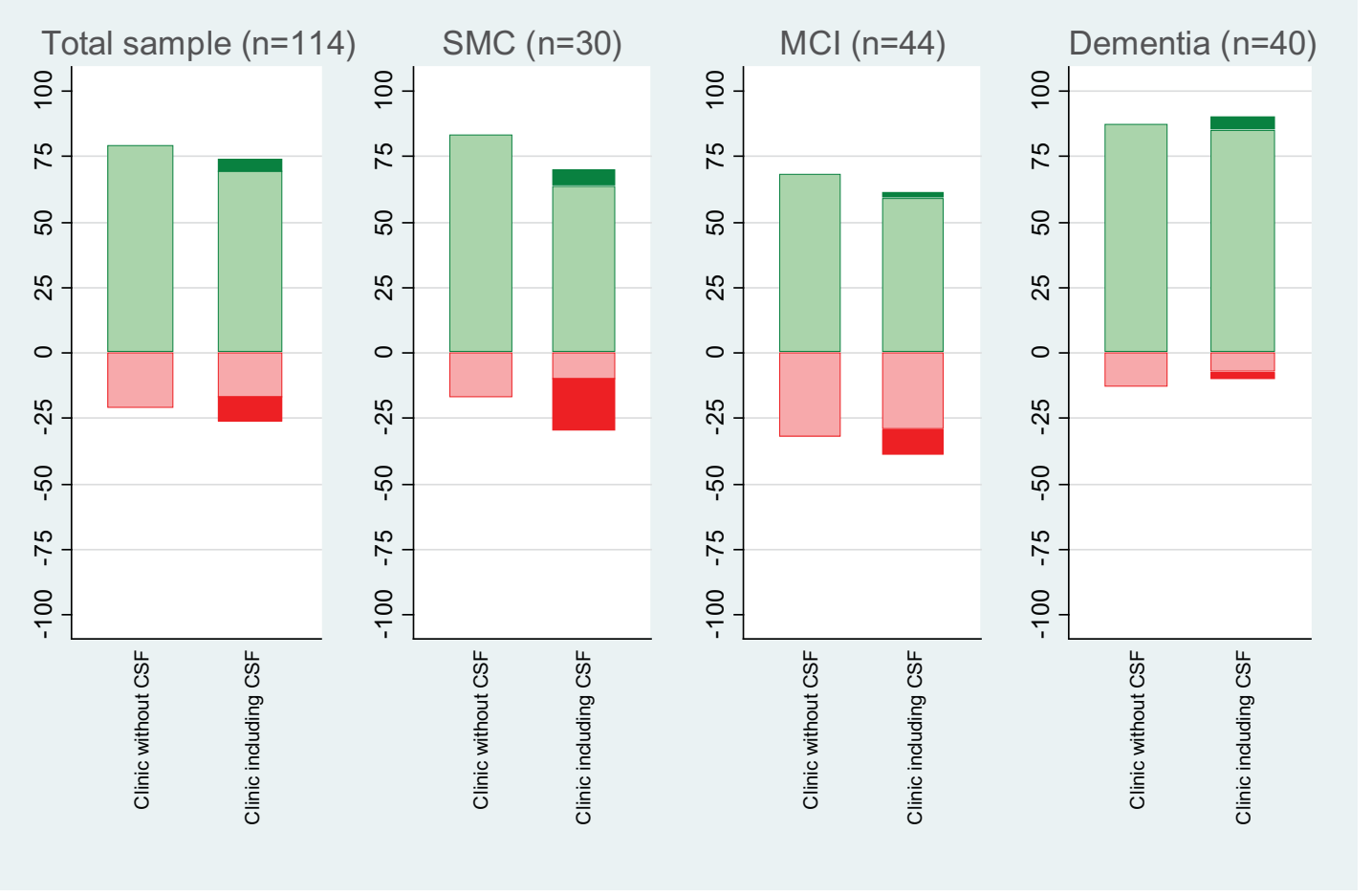

Fig. 3. Percentages (y-axis) correctly (light green shaded above 0) and incorrectly (light red shaded below 0) predicted based on the information from the "routine assessment without CSF", and percentages correctly (dark green shaded above 0) and incorrectly (dark red shaded below 0) differently predicted based on the information from the "routine assessment with CSF" of the total sample and the subsamples. The dark green and red shaded areas reflect the incremental diagnostic effect. SMC, subjective memory complaints; MCI, mild cognitive impairment.

a memory clinic. Although the numbers were small, knowledge of the CSF only improved prediction in those with established dementia, whereas it worsened prediction in those with SMC or MCI.

The lack of added prognostic value can be explained by the extensive information available in the standard practice routine diagnostic workup, which includes patient history, and physical, neurological, psychiatric, neuropsychological, and MRI examinations. This information appeared already sufficient for the clinical experts to predict the short-term course of symptoms fairly accurately. Another explanation may be the relatively short follow-up period. This may explain why in SMC and MCI participants the number of false-positive prognoses increased after use of CSF biomarkers since in these participants the expected clinical decline related to AD may take more than 2 years to become manifest $[29,30]$. Furthermore, the low pre-test probability of decline in SMC and high pre-test probability in dementia limits the room for improving the accuracy of predicted decline by CSF or any predictive test mainly to MCI participants.
Other studies that evaluated the predictive value of CSF biomarkers in addition to routine diagnostic tests in MCI patients found an added value compared to cognitive, neuropsychological, or MRI data $[6,7]$. These studies differed from ours as they had progression to dementia or decline on cognitive tests as an outcome measure, instead of clinical judgment of decline. Furthermore, they relied on a (logistic regression) prediction model to generate prognoses instead of expected decline by clinical judgment. We performed post-hoc analyses in our subsample of MCI patients using a logistic regression model similar to that used in these previous studies. We found an odds ratio of $2.9(p=0.256)$ for dementia conversion using the dichotomized CSF ratio (using the formula: CSF abnormal if $(\mathrm{A} \beta /(240+(1.18 *$ thau $)))<1[31])$, corrected for hippocampal volume, age and MMSE score. Furthermore, we found an improvement in classification of conversion to AD-type dementia in our subsample of MCI patients from $76 \%$ to $82 \%$ $(p=0.480)$ when using a logistic regression model similar to Ewers et al. [6] to classify each patient, consisting of the trail-making neuropsychological test 
and consisting of age, left manually scored hippocampal volume, and CSF ratio $A \beta / p t a u$ in addition to the trail-making test respectively. Although these effects were in the same direction as the previous studies, they were lower and not significant, possibly due to our limited sample size.

\section{Strengths and limitations}

This study's major strength is the extent to which actual practice was reflected by simulating clinical decision making regarding prognosis based on comprehensive test outcome descriptions of the study participants and discussion by a multidisciplinary panel of experts using a detailed pre-defined reference standard protocol. Nevertheless, it remains a simulation and simplifies actual practice. This applies in particular for clinical history taking, which was presented by short summaries. This could be improved using video recordings of the history taking.

The study was limited by the fact that the experts' assessments of observed decline (reference standard) took place immediately after the prediction of decline based on routine assessment without CSF biomarkers. This may have introduced diagnostic review bias, in favor of the routine assessment without CSF. Furthermore, the study could have suffered from incorporation bias, as the baseline diagnostic information from the routine assessment was available to the experts when they assigned the reference values. As a result the predictions based on the assessment without $\mathrm{CSF}$ and the assessment with CSF become more alike with the reference standard. However, the baseline test information can hardly be hidden when omitted for the reference standard, since a starting point of disease severity is required to rate the observed course of symptoms. Furthermore, this is unlikely to have resulted in a major bias, since the baseline information only provides a "piece of information" compared to the follow-up information provided to the experts [32] and it occurred both in the assessment without CSF as well as the assessment with CSF.

The information from the routine diagnostic workup in the patient descriptions (which was identical during the assessments without and with CSF information) may have been differently interpreted during the assessment with CSF biomarkers than in the assessment without CSF biomarkers, due to testretest variability. This might explain some of the differences in prognosis between the 2 assessments though does not introduce bias between the assessments and does not threaten the validity of the results.
Test review bias (could occur if the result of reference standard is known during the assessment with CSF information) was prevented by the 8.5-month period that elapsed between the two judgments.

While we used short-term prognosis as an outcome this would leave patients in a state of uncertainty after the period of 2 years. This underlines the importance of estimating the exact timing of an individual's expected course of decline over a longer follow-up period in future research and research into individual patient preferences.

This study was performed in specialized centers and the cohort represents a relative young population with a relatively low number of cases with $A D$ and high MMSE in the dementia subgroup. Therefore the results may not be generalizable to other settings. In the general population or general practices the prevalence of decline is most likely lower and the disease spectrum may differ. In an older population the prevalence of decline as well as positive CSF biomarkers is likely higher. The high MMSE in the dementia subgroup reflects a typical clinical population of mildly affected persons.

Although the experts were asked to establish the etiological diagnosis, and may have considered this in their decision-making process for predicting the course of symptoms, etiology was not a subject of evaluation in this study. Therefore, our results are not suitable for etiological diagnostic purposes such as treatment or trial participant selection.

Our conclusion that CSF biomarkers do not provide added value for prognosis is based on the assumption that the effect of informing a patient with a false positive and a false negative prognosis has a similar impact on his/her well-being. This assumption has not been tested, and may not be true. Possibly the favorable effect of the four participants whose prediction correctly changed from an expected course of decline to an expected stable/improved course might offset the unfavorable effect of the nine participants whose prediction incorrectly changed from an expected stable/improved course to an expected course of decline in the routine assessment with CSF biomarkers. Studies on the impact of an early prognosis on patient-relevant outcomes are therefore urgently needed to establish the clinical utility of AD biomarkers beyond diagnostic test accuracy outcomes [33].

\section{Implications for clinical practice}

This study evaluated the routine use of CSF biomarkers in Western-country academic memory 
clinics in which patients suspected for cognitive impairment are extensively evaluated. The lack of an added prognostic value implies that CSF should not be routinely used for short-term prognostic purposes in persons with memory complaints.

\section{ACKNOWLEDGMENTS}

This research was performed within the framework of CTMM, the Centre for Translational Molecular Medicine (http://www.ctmm.nl), project LEARN (grant $02 \mathrm{~N}-101$ ). The funding body had no role in the study design, the collection, analysis or interpretation of data, or the writing of the manuscript. The funding body checked the manuscript for possible infringement of intellectual property rights and approved the manuscript for publication without suggesting any revisions.

Authors' disclosures available online (http://www. j-alz.com/manuscript-disclosures/15-1120r1).

\section{SUPPLEMENTARY MATERIAL}

The supplementary material is available in the electronic version of this article: http://dx.doi.org/ 10.3233/JAD-151120.

\section{REFERENCES}

[1] Prince M, Bryce R, Ferri CP (2011) World Alzheimer Report 2011. Alzheimer Disease International, London.

[2] Sperling RA, Aisen PS, Beckett LA, Bennett DA, Craft S, Fagan AM, Iwatsubo T, Jack CR, Kaye J, Montine TJ, Park DC, Reiman EM, Rowe CC, Siemers E, Stern Y, Yaffe K, Carrillo MC, Thies B, Morrison-Bogorad M, Wagster MV, Phelps CH (2011) Toward defining the preclinical stages of Alzheimer's disease: Recommendations from the National Institute on Aging-Alzheimer's Association workgroups on diagnostic guidelines for Alzheimer's disease. Alzheimers Dement 7, 280-292.

[3] Albert MS, DeKosky ST, Dickson D, Dubois B, Feldman HH, Fox NC, Gamst A, Holtzman DM, Jagust WJ, Petersen RC, Snyder PJ, Carrillo MC, Thies B, Phelps CH (2011) The diagnosis of mild cognitive impairment due to Alzheimer's disease: Recommendations from the National Institute on Aging-Alzheimer's Association workgroups on diagnostic guidelines for Alzheimer's disease. Alzheimers Dement 7, 270-279.

[4] McKhann GM, Knopman DS, Chertkow H, Hyman BT, Jack CR, Kawas CH, Klunk WE, Koroshetz WJ, Manly JJ, Mayeux R, Mohs RC, Morris JC, Rossor MN, Scheltens P, Carrillo MC, Thies B, Weintraub S, Phelps $\mathrm{CH}$ (2011) The diagnosis of dementia due to Alzheimer's disease: Recommendations from the National Institute on Aging-Alzheimer's Association workgroups on diagnostic guidelines for Alzheimer's disease. Alzheimers Dement 7, 335-337.
[5] Vos S, van Rossum I, Burns L, Knol D, Scheltens P, Soininen H, Wahlund L-O, Hampel H, Tsolaki M, Minthon L, Handels R, L'Italien G, van der Flier W, Aalten P, Teunissen C, Barkhof F, Blennow K, Wolz R, Rueckert D, Verhey F, Visser PJ (2012) Test sequence of CSF and MRI biomarkers for prediction of $\mathrm{AD}$ in subjects with MCI. Neurobiol Aging 33, 2272-2281.

[6] Ewers M, Walsh C, Trojanowski JQ, Shaw LM, Petersen RC, Jack CR, Feldman HH, Bokde ALW, Alexander GE, Scheltens P, Vellas B, Dubois B, Weiner M, Hampel $\mathrm{H}$ (2012) Prediction of conversion from mild cognitive impairment to Alzheimer's disease dementia based upon biomarkers and neuropsychological test performance. Neurobiol Aging 33, 1203-1214.e2.

[7] Richard E, Schmand BA, Eikelenboom P, Van Gool WA (2013) MRI and cerebrospinal fluid biomarkers for predicting progression to Alzheimer's disease in patients with mild cognitive impairment: A diagnostic accuracy study. BMJ Open 3, pii: e002541.

[8] Palmqvist S, Hertze J, Minthon L, Wattmo C, Zetterberg H, Blennow K, Londos E, Hansson O (2012) Comparison of brief cognitive tests and CSF biomarkers in predicting Alzheimer's disease in mild cognitive impairment: Six-year follow-up study. PLoS One 7, e38639.

[9] Heister D, Brewer JB, Magda S, Blennow K, McEvoy LK (2011) Predicting MCI outcome with clinically available MRI and CSF biomarkers. Neurology 77, 1619-1628.

[10] Handels RLH, Aalten P, Wolfs CAG, OldeRikkert M, Scheltens P, Visser PJ, Joore MA, Severens JL, Verhey FRJ (2012) Diagnostic and economic evaluation of new biomarkers for Alzheimer's disease: The research protocol of a prospective cohort study. BMC Neurol 12, 72.

[11] Cushman L, Scherer MJ (1995) Psychological assessment in medical rehabilitation, American Psychological Association, Washington, DC.

[12] Morris JC (1993) The Clinical Dementia Rating (CDR): Current version and scoring rules. Neurology 43, 24122414.

[13] Hughes CP, Berg L, Danziger WL, Coben LA, Martin RL (1982) A new clinical scale for the staging of dementia. $\mathrm{Br}$ J Psychiatry 140, 566-572.

[14] Almeida OP, Almeida SA (1999) Short versions of the geriatric depression scale: A study of their validity for the diagnosis of a major depressive episode according to ICD-10 and DSM-IV. Int $J$ Geriatr Psychiatry 14, 858-865.

[15] Cummings JL (1997) The Neuropsychiatric Inventory: Assessing psychopathology in dementia patients. Neurology 48, S10-S16.

[16] Gélinas I, Gauthier L, McIntyre M, Gauthier S (1999) Development of a functional measure for persons with Alzheimer's disease: The disability assessment for dementia. Am J Occup Ther 53, 471-481.

[17] Rey A (1958) L' examen clinique en psychologie, Presses universitaires de France, Oxford, England.

[18] Brand N, Jolles J (1985) Learning and retrieval rate of words presented auditorily and visually. J Gen Psychol 112, 201210.

[19] Lindeboom J, Schmand B, Tulner L, Walstra G, Jonker C (2002) Visual association test to detect early dementia of the Alzheimer type. J Neurol Neurosurg Psychiatry 73, 126133.

[20] Wechsler D (1997) WMS-III: Wechsler Memory Scale Administration and Scoring Manual, Psychological Corporation. 
[21] Natu M V, Agarwal AK (1995) Digit Letter Substitution Test (DLST) as an alternative to Digit Symbol Substitution Test (DSST). Hum Psychopharmacol Clin Exp 10, 339-343.

[22] Stroop JR (1935) Studies of interference in serial verbal reactions. J Exp Psychol 18, 643-662.

[23] Reitan R (1956) Trail Making Test: Manual for Administration, Scoring and Interpretation., Section of Neuropsychology, Department of Neurology, Indiana University Medical Centre, Bloomington.

[24] Reitan RM (1958) Validity of the Trail Making Test as an indicator of organic brain damage. Percept Mot Ski 8, 271276.

[25] Scheltens P, Leys D, Barkhof F, Huglo D, Weinstein HC, Vermersch P, Kuiper M, Steinling M, Wolters EC, Valk J (1992) Atrophy of medial temporal lobes on MRI in "probable" Alzheimer's disease and normal ageing: Diagnostic value and neuropsychological correlates. J Neurol Neurosurg Psychiatry 55, 967-72.

[26] Fazekas F, Kleinert R, Offenbacher H, Schmidt R, Kleinert G, Payer F, Radner H, Lechner H (1993) Pathologic correlates of incidental MRI white matter signal hyperintensities. Neurology 43, 1683-1689.

[27] Handels RLH, Wolfs CAG, Aalten P, Bossuyt PMM, Joore MA, Leentjens AFG, Severens JL, Verhey FRJ (2014) Optimizing the use of expert panel reference diagnoses in diagnostic studies of multidimensional syndromes. $B M C$ Neurol 14, 190.

[28] Bertens LCM, Broekhuizen BDL, Naaktgeboren CA, Rutten FH, Hoes AW, van Mourik Y, Moons KGM, Reitsma JB
(2013) Use of expert panels to define the reference standard in diagnostic research: A systematic review of published methods and reporting. PLoS Med 10, e1001531.

[29] Visser PJ, Verhey F, Knol DL, Scheltens P, Wahlund L-O, Freund-Levi Y, Tsolaki M, Minthon L, Wallin AK, Hampel H, Bürger K, Pirttila T, Soininen H, Rikkert MO, Verbeek MM, Spiru L, Blennow K (2009) Prevalence and prognostic value of CSF markers of Alzheimer's disease pathology in patients with subjective cognitive impairment or mild cognitive impairment in the DESCRIPA study: A prospective cohort study. Lancet Neurol 8, 619-627.

[30] Bertens D, Knol DL, Scheltens P, Visser PJ (2015) Temporal evolution of biomarkers and cognitive markers in the asymptomatic, MCI, and dementia stage of Alzheimer's disease. Alzheimers Dement 11, 511-522.

[31] Hulstaert F, Blennow K, Ivanoiu A, Schoonderwaldt HC, Riemenschneider M, De Deyn PP, Bancher C, Cras P, Wiltfang J, Mehta PD, Iqbal K, Pottel H, Vanmechelen E, Vanderstichele H (1999) Improved discrimination of AD patients using beta-amyloid(1-42) and tau levels in CSF. Neurology 52, 1555-1562.

[32] Moons KGM, Grobbee DE (2002) When should we remain blind and when should our eyes remain open in diagnostic studies? J Clin Epidemiol 55, 633-636.

[33] Ferrante di Ruffano L, Hyde CJ, McCaffery KJ, Bossuyt PMM, Deeks JJ (2012) Assessing the value of diagnostic tests: A framework for designing and evaluating trials. $B M J$ 344, e686. 